\title{
The Strömgren Automatic Telescope
}

\author{
Ralph Florentin-Nielsen
}

\author{
Copenhagen University Observatory
}

\begin{abstract}
Copenhagen University Observatory operates a $52 \mathrm{~cm}$ Cassegrain telescope at ESO, La Silla (Chile) for photoelectric photometry in a completely automatic mode. Alternatively, the telescope may be operated, remotely, from Europe. We report on the operational experience from more than four years of successful use and on the decision by ESO to establish a similar system on their photometric telescope.
\end{abstract}

\section{The telescope and Instrumentation}

The Strömgren Automatic Telescope (SAT) is a 52-cm Cassegrain telescope. The optics are a Dahl-Kirkham design with an ellipsoidal primary and a spherical secondary. The telescope is operated by Copenhagen University Observatory at the European Southern Observatory, La Silla, Chile. It is used solely for stellar photoelectric photometry. A special purpose spectrophotometer (R. Florentin-Nielsen, 1985 and Helt, Franco and Florentin-Nielsen, 1987) for Strömgren uvby, H photometry is permanently mounted at the SAT. This spectrophotometer uses a reflection grating in Littrow configuration. The uvby spectral bands are defined by interference filters in conjunction with the output slots. The filters have very high transmission mainly due to the fact that the spectrograph slots removes the need for blocking for side bands.

The telescope is used mainly for the observation of eclipsing binaries, Andersen \& Clausen, 1989 and for large scale classification work, Gray and Olsen, 1991 and references therein. Simultaneous photometry and radial velocities measurements done with the Coravel instrument at the Danish 1.54-m telescope at La Silla have yielded absolute dimensions of eclipsing binaries. A review paper by Andersen, 1991, shows that about $60 \%$ of all 45 systems with radii and masses known with an accuracy to 1 - 2 percent have been determined from the Danish observations.

The telescope was equipped with a computer controlled six channel photon counting system, Klougart, 1984a and a digital telescope drive system, Klougart, 1984b. However, due to the very large amount of photometry carried out with that telescope it was decided to make the observations completely automatic. The telescope drive and control system already computes the apparent places for the stars to be observed and applies a pointing model correction (look-up table in declination and hour angle) before setting the telescope. Also the photon counting systems have built-in routines to test the quality of the photometry by comparing the actual spread of multiple 1 
second integrations with the spread expected from the photon shot noise alone. Finally, the photon counting system may be set to either use specified integration times, to integrate until a required photometric accuracy is obtained or a combination of the two methods, Claudius and Florentin-Nielsen, 1981.

What remained to be established to allow an automatic observing mode was a method to center the telescope at the stars and a program to let the telescope control system and the photon counting system communicate with each other and to arrange for the proper sequence of the observations.

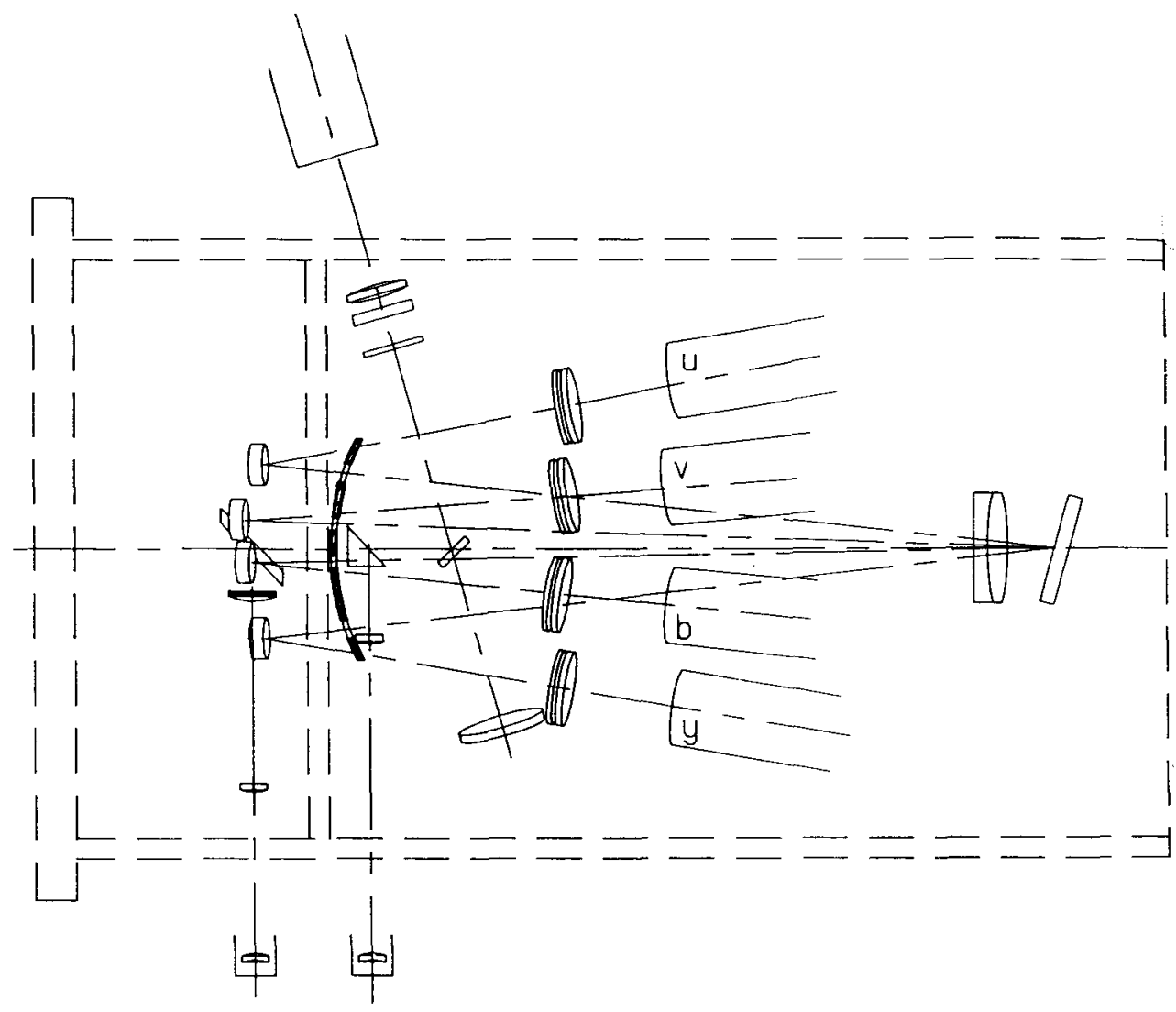

Figure 1a. The optical layout of the 6-channel uvby, $\mathrm{H} \beta$ spectrophotometer of the Strömgren Automatic Telescope. 


\section{The Auto-Centering Device}

We have chosen not to incorporate any additional two dimensional detector (CCD) to the existing spectrophotometer. The setting error of the telescope is determined by scanning the star across a $\mathrm{V}$-shaped slit plate in the focal plane. As can be seen from figure 3 the V-shaped slits are formed directly in one of the positions of the focal plane aperture wheel. The transits of the star over the slits are detected as the sum of the signals from the photomultipliers for all four channels, $\mathrm{u}, \mathrm{v}, \mathrm{b}$ and $\mathrm{y}$.

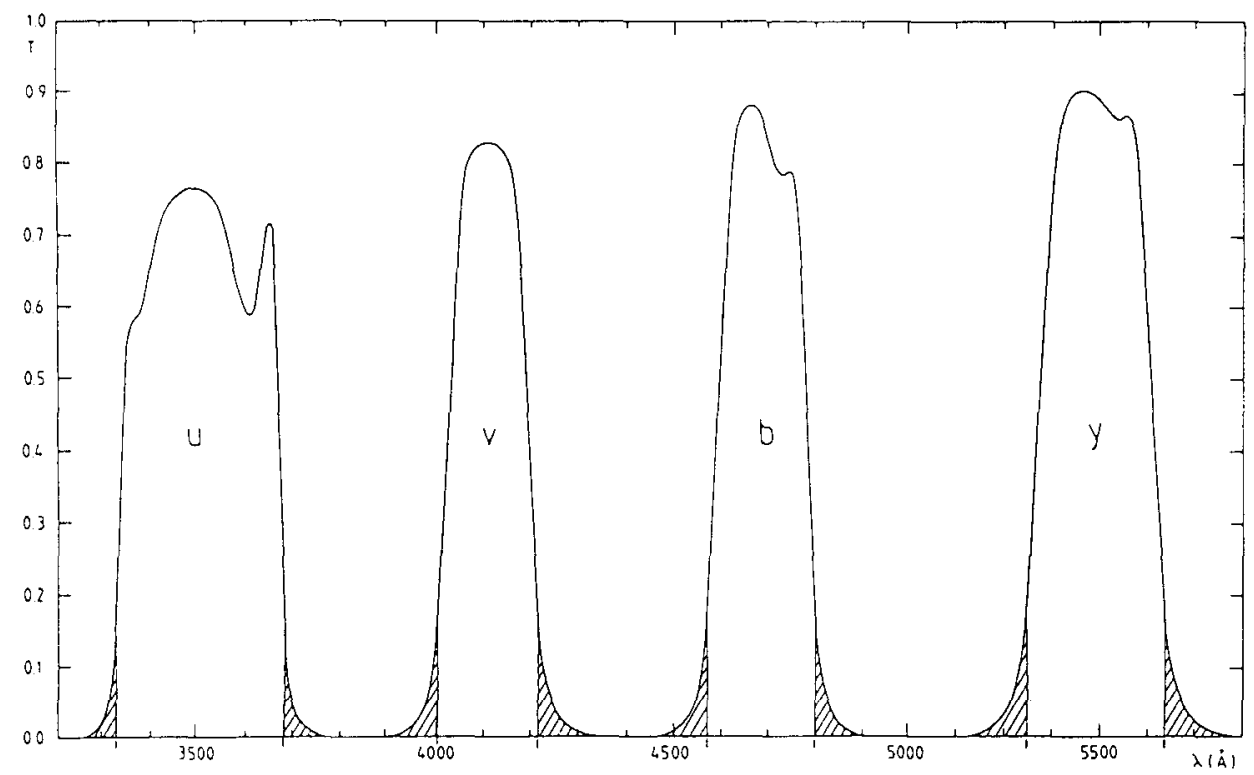

Figure 1b. The optical transmission of the $u, v, b$ and $y$ filters. Shaded areas of the passbands are blocked by the output slot section of the spectrograph.

A preset to a new star to be observed is then performed by setting the telescope to a position which is offset by 2 arcminutes in declination, while at the same time turning the aperture wheel to the $\mathrm{V}$-slit position, immediately followed by a linear scan in delta. The first 60 arcsec is utilized to settle the servos to a constant scanning speed of $40 \mathrm{arcsec} / \mathrm{second}$. During the subsequent $60 \mathrm{arcsec}$ the sum of the photon counts from the 4 photomultipliers are sampled every 50 millisec. The double-peaked intensity profile obtained over this 60 arcsec ( 1.5 seconds) scan is analysed to calculate the setting errors in RA and Dec, $\delta \alpha$ and $\delta \delta$. The times for the median of the peaks from the transit of the upper and lower part of the $\mathrm{V}$-slit are found by numerical convolution of the scan data with a line spread function, and are denoted $t_{1}$ and $t_{2}$. The start time of the $60 \operatorname{arcsec} s c a n$ is denoted $t_{0}$. Refer figure 3 . It can be seen that the distance in time between the two transits $t_{1}$ and $t_{2}$ is a measure of the setting 
error in RA, whereas $t_{1}-t_{0}$ and $t_{2}-t_{0}$ defines the setting error in declination.

$$
\begin{gathered}
\delta \alpha=-\mathrm{v}(\operatorname{scan}) / 2 *\left[\left(t_{2}-t_{1}\right)-\left(t_{2}^{\prime}-t_{1}^{\prime}\right)\right] \\
\delta \delta=\mathrm{v}(\operatorname{scan}) *\left[\left(t_{1}+t_{2}\right) / 2-t_{0}-\left(t_{1}^{\prime}+t_{2}^{\prime}\right) / 2-t_{0}^{\prime}\right]
\end{gathered}
$$

where the scanning speed, $\mathrm{v}(\mathrm{scan})$ is $40 \mathrm{arcsec} / \mathrm{sec}$, and $t_{0}^{\prime}, t_{1}^{\prime}$ and $t_{2}^{\prime}$ are the times for the scan start and the two transits for a star which is perfectly centered $(\delta \alpha=\delta \delta=0)$. For the scan start time, $t_{0}$ defined as $0 \mathrm{sec}$ the nominal values of $t_{1}^{\prime}$ and $t_{2}^{\prime}$ are 375 and $1125 \mathrm{msec}$ respectively.

Immediately following the search scan the position errors are calculated and the telescope sets to the corrected position, and the aperture wheel is turned to the desired circular focal plane diaphragm. The total autocentering procedure takes slightly less than 5 seconds. The autocentering device works reliably down to a limiting magnitude of $11 \mathrm{~m}$ in uncrowded fields. In cases, where positions of the program stars are not known very accurately the autocentering may perform multiple, longer scans parallel to each other. This, however, is quite wasteful in terms of time, and

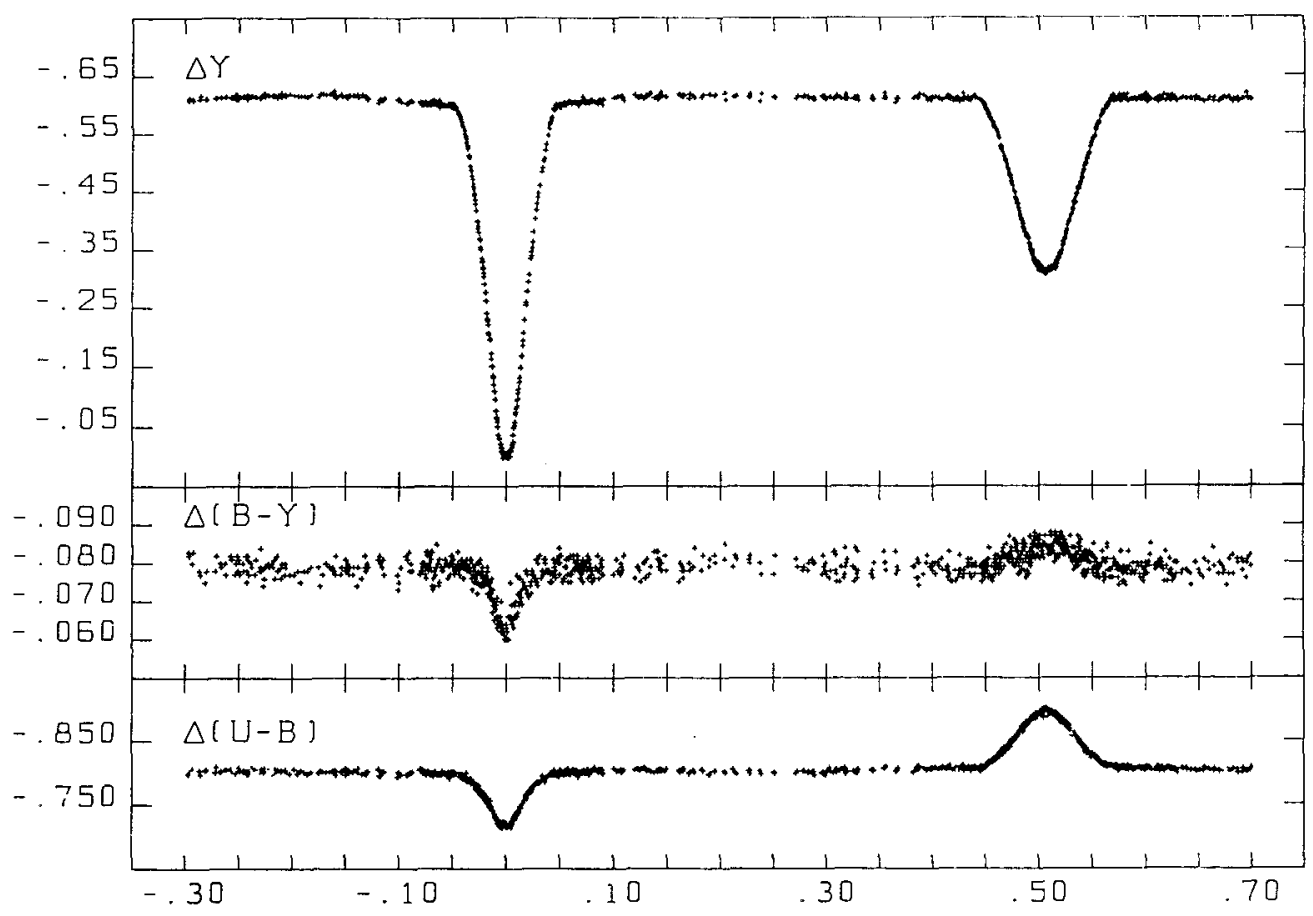

Figure 2. Light and colour curves for GG Lup observed at SAT by Anderson, Clausen and Giminéz (not yet published). Magnitude differences are accurate to 0.004 mag rms. 


\section{APERTURE WHEEL, S.A.T.}

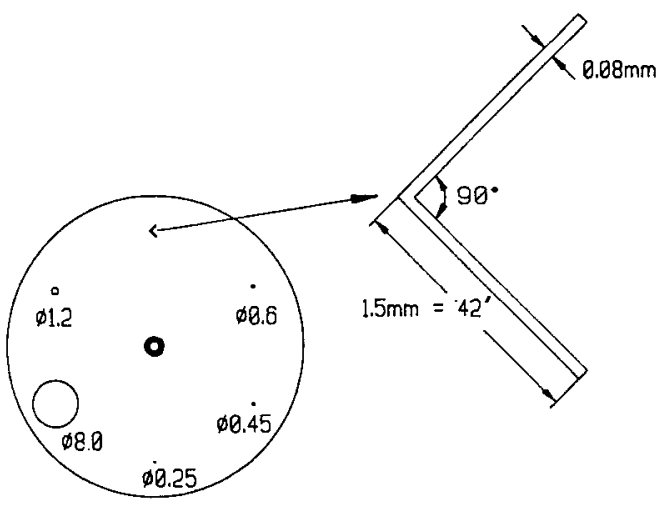

SEARCH SCAN, S.A.T
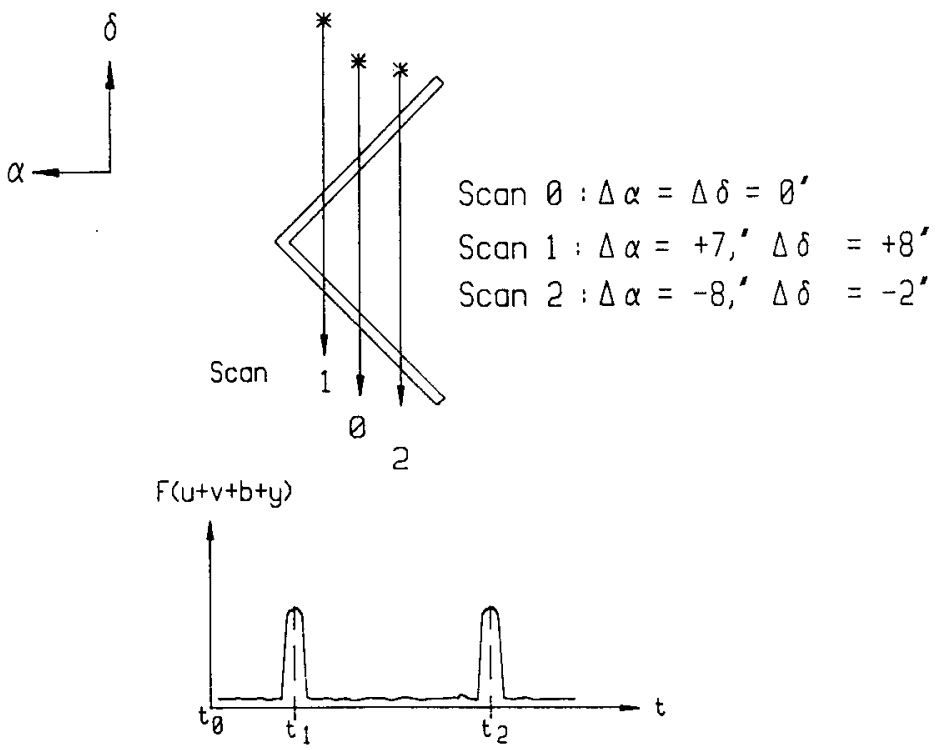

Figure 3. The focal plane aperture wheel of SAT with an enlarged view of the V-shaped slits. The star to be centred is scanned across the slits. The lower half of this figure shows three search scans. Scan 0 is for a star which was perfectly centred. Scans 1 and 2 have setting errors in RA and Dec of +7 and $+8 \operatorname{arcsec}(\operatorname{scan} 1)$ and -8 and $-2 \operatorname{arcsec}(\operatorname{scan} 2)$. Setting errors of the telescope are calculated from the profiles of the sum of the $u, v, b$ and y channels. 
hence to maintain telescope efficiency observers are always encouraged to prepare good positions, including proper motions if available. The observing rate for stars brighter than 9th magnitude is typically 30 stars per hour.

If a search field is crowded by other stars, or if the scan profiles indicate that the star is double, then the telescope will make no attempt to observe that star, but leave a mark in the observing list indicating that this star need to be inspected and set at manually. If the separation of a double star is less than about two arcsec (the limit depends somewhat on the difference in magnitude of the two components), then the telescope will set on the center of gravity of the flux from the two components.

\section{Automatic Sequencing of the Observations}

To operate automatically the system needs at least three files stored on hard disk. They are: 1) An observing list or star catalogue with the positions, equinoctium and proper motions. 2) An output file to store results, error messages and comments by the observer. 3) A command file containing a string of instructions on how the observations should be carried out. The most important instruction is OBS, which will preset the telescope, start a search scan, autocenter the telescope, select the diaphragm size, select the uvby or $\beta$ section and initiate an integration sequence. Following the OBS instruction a record number specifies the star from the star catalogue. Alternatively OBS $\mathrm{N}$ sets on the next star in the catalogue and observes it. A jump (JMP) instruction can be used to repeat the observations of a number of stars (e.g. variable, comparison and check star), for example until a specified sidereal time.

All functions of controlling the telescope, dome, auto-centering, and data acquisition from the 6 channel photon counter, observing sequencer and data logging on the SAT is governed by one VME-10 computer. Nörregaard, 1988.

The system has been continuously in operation since 1987 (Florentin-Nielsen, Nörregaard, Olsen, 1987). Besides occasional problems with a disk drive and dome rotation the system has been remarkably reliable. Over the last four years no observing night has been lost due to technical reasons.

\section{Operation in the immediate future}

Transport of observing lists, command files and observational data is done on VME diskettes. As several user institutes have no access to read or write VME diskettes, it has been decided to interface a PC AT to the VME computer. (European astronomers generally tend to prefer PCs for most of their work). Not only can data then be transported on PC diskettes, but also the observers may add extra PC software to do on-line data reductions or display light curves of variables, show extinction plots or any other program that the observer may find useful. Some such data reduction PC software already exists, but it is the general policy that while individual observers may not modify the VME software, they may add as much PC software as they like.

In practical use observers may attend the observations from the office downstairs to watch astronomically relevant data being created or displayed, or just occasionally 
verify that the quality of the photometry is good. Several Danish observers have had telescope time at the SAT and at the Danish 1.54-m telescope at La Silla at the same time, thereby saving travelling expenses.

ESO has this year decided to have their 50 -cm photometric telescope rebuilt, such that it will become a close copy of the SAT. The ESO $50-\mathrm{cm}$ has an ordinary one channel filter photometer. This upgrade is presently been made (mechanics at ESO, electronics and software in Denmark), and is planned to go in operation in February 1993. After that time ESO will not generally offer travel grants for observers to go to the SAT or ESO 50-cm telescope. One ESO night assistant will load the observing list into the VME and start the observing procedure in the beginning of night. All data transport between Europe and Chile will be by PC diskettes or electronic mail.

\section{References:}

Andersen, J.: 1991, The Astron. Astrophys. Rev. 3, 91.

Andersen, J., Clausen, J.V.: 1989, Astron. Astrophys. 213, 183.

Claudius M., Florentin-Nielsen, R.: 1981, Astron. Astrophys. 100, 186.

Florentin-Nielsen, R.: 1985, A 6-channel uvby-beta Spectrophotometer for the Danish 50 cm Telescope, Copenhagen Univ. Obs. Internal Report 8, 1.

Florentin-Nielsen, R., Nörregaard, P., Olsen, E.H.: 1987, First Fully Automatic Telescope at La Silla, ESO Messenger, No. 50, 45.

Gray, R.O., Olsen, E.H.: 1991, Astron. Astrophys. Suppl. Ser. 87, 541.

Helt, B.E., Franco, G.A.P., Florentin-Nielsen R.: 1987, Proc. ESO Workshop on SN $1987 \mathrm{a}, 89$.

Klougart, J.: 1984a, Photon Counting System (Photsys) Danish $50 \mathrm{~cm}$, Users manual, 1. Klougart, J.: 1984b, Telescope Control System, Users manual, 1.

Nörregaard, P.: 1988, Control System on Strömgren Automatic Telescope, Version 3.0, Users manual, 1.

\section{Discussion}

R.R. Shobbrook: How are sky positions chosen? Highest accuracy would be attained by choosing a position from a deep photograph.

Florentin-Nielsen It is certainly true that observers working in clusters or crowded fields have been selecting their sky background positions carefully from maps. However, much of the work on bright stars in uncrowded fields has been done using a nominal position offset, and then verifying that this position was alright.

C.L. Sterken: We did long-term monitoring during the manual lifetime of the telescope, and also after automation. We indeed found that we could gain more than a millimagnitude precision by replacing the sky offset selected previously by visual inspection, by sky positions selected from sky photographs. 
When observing in NGC3293, applying a three-star and sky sequence during 5 or 6 hours, I experienced more than once that the system would centre all my stars during several hours, but it would happen once in a while that the system would fail, and search endlessly for the star it had previously been able to find without problem. This happens about once in 3-4 nights. Do you have any clue as to what may be at the origin of this problem?

Florentin-Nielsen: This has probably been a software bug. It is a large control programme, and several corrections and additions have been made to the software.

A. T. Young: How accurately does your pointing model find stars?

Florentin-Nielsen: The pointing accuracy is 5-10 arc sec over the sky.

D.L. Crawford: Can you comment on the stability of the photometric system over the years (as the hardware has been very stable over a long period)?

Florentin-Nielsen: The instrumental system has been remarkably stable over the years, so much so, in fact, that variable star observers have had no need to transform to the standard system for stability reasons. 